portant that the physiotherapist should supervise the movements and make sure that the metacarpal is again moved through a full active range at the new joint.

This treatment is continued until the patient can return to light work, which is usually a matter of four to six weeks. Some patients complain of pain for longer than this, but others are surprisingly pain free; one started milking cows within a month of operation.

\section{Results and Case Reports}

All the patients have been very satisfied with the results of the operation, and have reported improved function and little or no pain. Removal of the trapezium leaves the thumb a little weaker than the normal, but a full range of movement of the metacarpal is regained with no obvious deformity.

Mr. E. B., aged 48, farm worker. Pain and swelling at the base of the right thumb which he dated from an injury two years before. The right trapezium was excised in April, 1944. He worked full time after this, but attended again in 1947, complaining of pain in the left thumb (Fig. 2). The left trapezium was excised in February, I947. He was able to do full work inside two months and has been doing full farm work since. He states there is no pain and he can do anything. On examination in January, 1948, there was a full and painless range of movement with good power in both thumbs, but slight crepitus on movement on the right side (Fig. 4).
Mrs. G. F., aged 53, housewife. The right anc left bones were excised in November, 1945 (Fig. I)은 She is now very satisfied and has no pain, but states that her hands are not quite so strong for turning taps as previously. The radiographs: taken in January, I948, show a satisfactory resultw on the right, but on the left a fragment of theo trapezium remains in situ (Fig. 5). It is interesting to note how this fragment has increased inc. size since the film taken one year after operation (Fig. 6).

Miss $E$. W., aged 46 , sempstress. Four years of pain and difficulty in carrying out her work. The $\overrightarrow{0}$ right trapezium was excised in April, 1944. She $\vec{\omega}$ has been working full time since with no pain.

Miss $N$., aged 58 . Symptoms following an in jury nine months before. Pain on using. left thumb and a marked cystic swelling over thid trapezio-metacarpal joint (Fig. 3). The trapezium was excised in April, 1944. In 1948 she reported 2 that her thumb was quite satisfactory and shei was very pleased that she had undergone the operation.

$M r . Q . W$. , aged 62, retired, is an example of the results in older patients. He gave a history of pain over a period of five years. with unsuccessfuk treatment by physiotherapy. The right trapezium $\overrightarrow{0}$ was excised in February, 1947. He now amu@ himself sawing up logs.

\section{BIBLIOGRAPHY}

GERVIS, W. H. (1947), Pròc. Roy. Soc. Med., 40; 492.

RAY, M. B. (1933), Proc. Roy. Soc. Med., 27, 193.

FORESTIER, J. (1927), La Presse Medicale.

\title{
MEDIAN NERVE COMPRESSION IN THE CARPAL TUNNEL
}

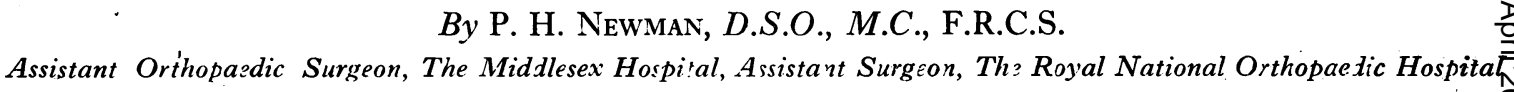

The carpal tunnel is a very confined space. It has been designed as a trough with a hard floor and rigid bony walls in order to protect the important structures within its confines. But for this anatomical arrangement, it is unlikely that the flexor tendons with their delicate sheaths and the median nerve could withstand the trauma of recurrent falls on the outstretched hand. The walls, which consist on the the medial side of the pisiform and the hook of the hamate, and on the latera side, of the tubercle of the scaphoid and the ridges on the trapezium, have stretched across between their peaks a fibrous sheet forming a roof knowre as the transverse carpal ligament. This is a tough structure giving additional protection and acting. as a bar to forward prolapse of the structures when the wrist is in flexion.

The tendons and nerve pass from the loose en- 
vironment of the soft tissues of the forearm through this bottleneck into the comparative freedom of the palm. As the price of protection from exposure to danger at this point, they forfeit as it were their liberty of expansion and freedom of movement other than that which is essential for the function of the hand and fingers. There is no place for an abnormal body or for any hypertrophic process within this tunnel.

Acute compression of the median nerve at this level occurs from forward dislocation of the lunate bone (Fig. I), and may occasionally occur in other injuries of the carpus with displacement. In a comminuted Colles' fracture a fine spicule of bone may project forwards between the flexor tendons and impinge directly on the median nerve. A more insiduous onset of symptoms due to compression may occur as a result of hypertrophic arthritis of the wrist as from an old fracture of the scaphoid (Fig. 2), or even from a malunited Colles' fracture. In these cases there is some process which takes up space within the tunnel and undue pressure falls upon the normal contents. The tendons derive some protection from their sheaths and do not register symptoms of lateral compression unless it be severe. The nerve has no such sheath, is anchored laterally and is susceptible to alteration in pressure.

Denny-Brown and Brenner(1944) in two articles have given the results of experiments in which different degrees of pressure were applied to peripheral nerves. They found that the effect on conductivity of the nerve varies considerably according to the degree of pressure and the length of time during which it is applied. The pressure plate was I I mm. in length. In one series of experiments, if a light pressure of 5 to $7 \mathrm{gm}$. was applied continuously there was no interference of conduction, but narrowing of the nerve occurred at the site of pressure with swelling above and below the site of pressure. An increased pressure of 9 to $10 \mathrm{gm}$. was attended with impairment of motor function within six days and progressed to complete paralysis shortly afterwards. If, however, the pressure was continued, the nerve overcame this critical stage and function returned slowly. At the site of pressure, ischaemic and degenerative changes took place, but there was no nerve or muscle degeneration distal to this area.

Continued pressure for several days of $44 \mathrm{gm}$. was followed by complete loss of all conductivity and degeneration of the nerve both distally and for some distance proximally. Release of pressure was not followed by return of function. Compression by much greater pressure, e.g. $430 \mathrm{gm}$., for only two hours, was followed by recovery in about two weeks. They came to the conclusion that all the changes found were of an ischaemic rather than of a directly traumatic nature. Briefly speaking the changes in order of severity are :

I. Oedema of nerve without loss of conductivity.

2. Transient paralysis with spontaneous recovery.

3. More severe loss of function with slow recovery after release of pressure.

4. Complete loss of conductivity without the possibility of recovery, due to total nerve degeneration.

Recently Brain, Wright and Wilkinson (1947) have described symptoms of carpal tunnel compression of the median nerve arising spontaneously in six patients. In no case was there any obvious encroachment upon the space of the tunnel. The patients were all women in the second half of life and five were occupied in household duties.

Experimentally a tambour was placed in the carpal tunnel of a cadaver and it was found that the tension was three times greater when the wrist was extended to a right angle than when flexed to the same degree. They point out that the duties of a housewife necessitates much work with the wrist in the extended position and they consider that this, combined with some degeneration in the artery of the nerve, is responsible for the commencement of a vicious circle of oedema and increasing tension.

\section{Glinical Types}

Clinically three types of compression of the median nerve in the carpal tunnel are found. Firstly, an acute traumatic type associated with injuries such as anterior dislocation of the lunate bone ; the symptoms recover with replacement of bone, provided that this is not delayed too long. Secondly, slow compression of the nerve of spontaneous onset. Thirdly, slow compression secondary to malunion or arthritis in the region.

\section{Symptoms and Signs of Slow Compression}

The symptoms are generally those of weakness of the hand and loss of the power to grip, especially with the thumb. There may be a history of dropping such articles as a cup of tea or saucepan. Tingling or pins and needles are felt over the median nerve distribution. Pain is common and extends from the thumb or index finger below, to the forearm, upper arm or even to the neck above ; it is worse at night and aggravated by carrying heavy objects. It is sometimes relieved by working the fingers and wrist backwards and forwards.

The following signs may be found in cases of this sort :

r. Wasting of the thenar eminence, especially the outer half.

2. Sensory loss in the median area. 


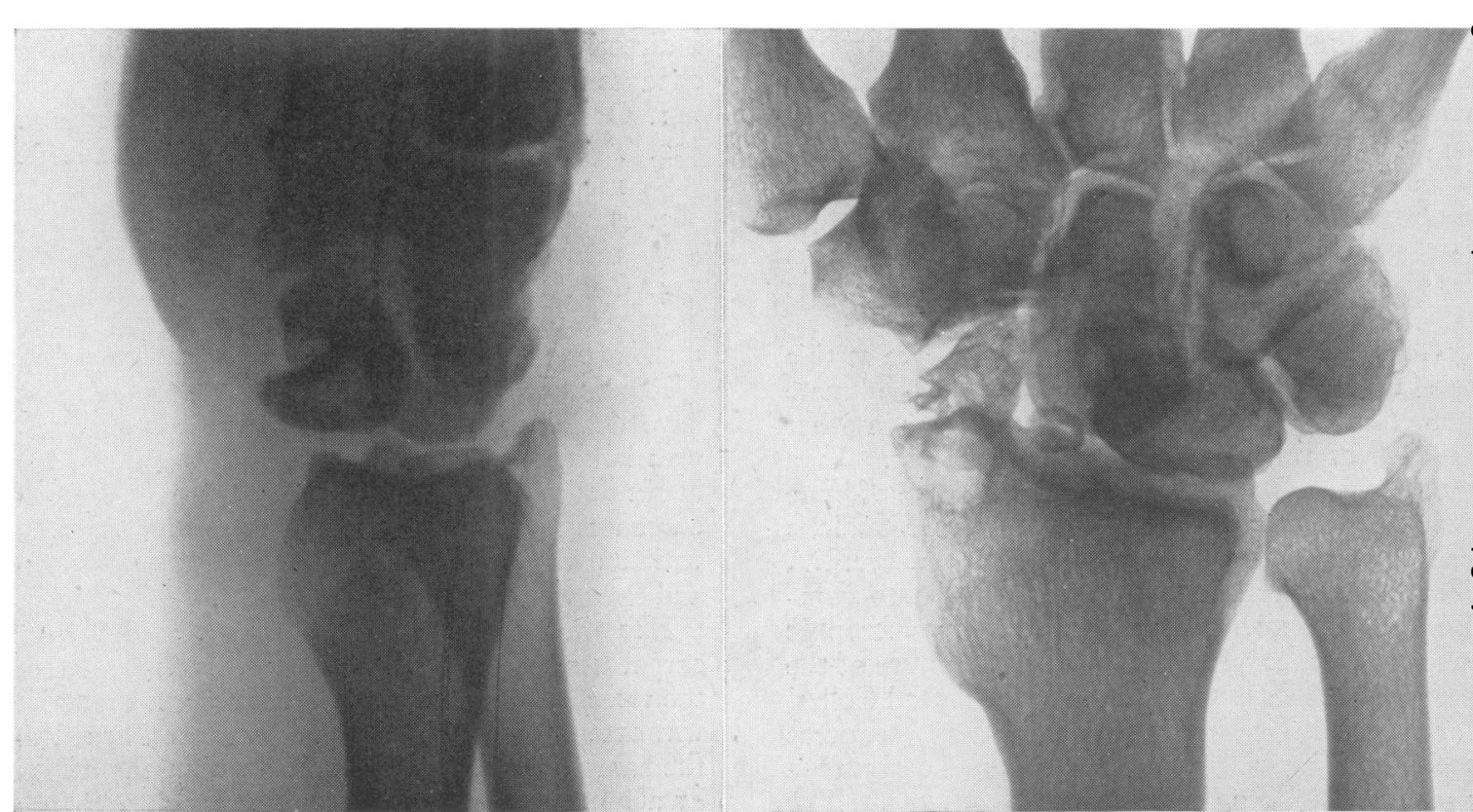

FIG. 1.-Forward dislocation of the lunate bone associated with median nerve palsy.

3. A localized swelling proximal to the transverse ligament.

4. Loss of power of extension in the outer three digits.

5. Impaired movement of the wrist and radiological changes, only in the secondary type of slow compression.

Wasting of the thenar eminence occurs typically in the Abductor Pollicis Brevis and Opponens. It may occur in the Flexor Pollicis Brevis, as the nerve supply of this muscle varies. Highet (1943), studying war wounds of the median nerve, found that the Flexor Brevis is supplied four times in five by the ulnar nerve. The two lateral lumbricals are also affected, but this paresis is very difficult to detect clinically.

Sensory impairment can often be detected over the distal phalanges and includes light touch and discrimination, temperature and pain. More extensive sensory loss may occur.

The swelling proximal to the transverse ligament is sometimes very obvious and may resemble a compound palmar ganglion. It is, however, more localized and has no fluid thrill. The swelling in the palm is negligible.

Loss of extension of the thumb, index or middle. fingers has been reported in some cases. This may be explained by the paralysis of the lateral two lumbricals or of the Flexor Pollicis Brevis.
FIG. 2.-Radio-carpal arthritis secondary to old fracture $\vec{z}$ of the right scaphoid (Patient C).

The latter is a synergist with the Extensor Pollic

Longus.

\section{Treatment}

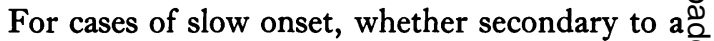
space-occupying lesion process or of apparently $\underset{\complement}{\complement}$ spontaneous origin, incision of the transverse liga- $\overrightarrow{\vec{\theta}}$ ment is a rational procedure. A vertical midline 3 incision is made, extending no further than the proximal boundary of the transverse ligament to: avoid placing the scar on the important pressure area in front of the carpus. As soon as the in 3 . cision is made the median nerve is generally at once obvious, grossly swollen and prolapsed. ventrally to lie immediately beneath the deepᄋ fascia. The distal end of the skin incision is lifted up and the transverse ligament incised음 subcutaneously in a vertical direction. The $>$ nerve beneath the ligament is compressed, in marked contrast with the swollen proximal o segment.

\section{Differential Diagnosis}

The conditions which may give rise to confusiono do so because they cause pain, wasting, or localo swelling. The fact that the primary type of com- $\frac{\bar{D}}{\mathrm{D}}$ pression occurs as a bilateral condition in middle $\stackrel{\mathscr{P}}{+}$ aged women doing heavy housework helps to 0 eliminate some of these condition. The secondary type : shows radiological signs of old injury or arthritic changes and is generally unilateral. 


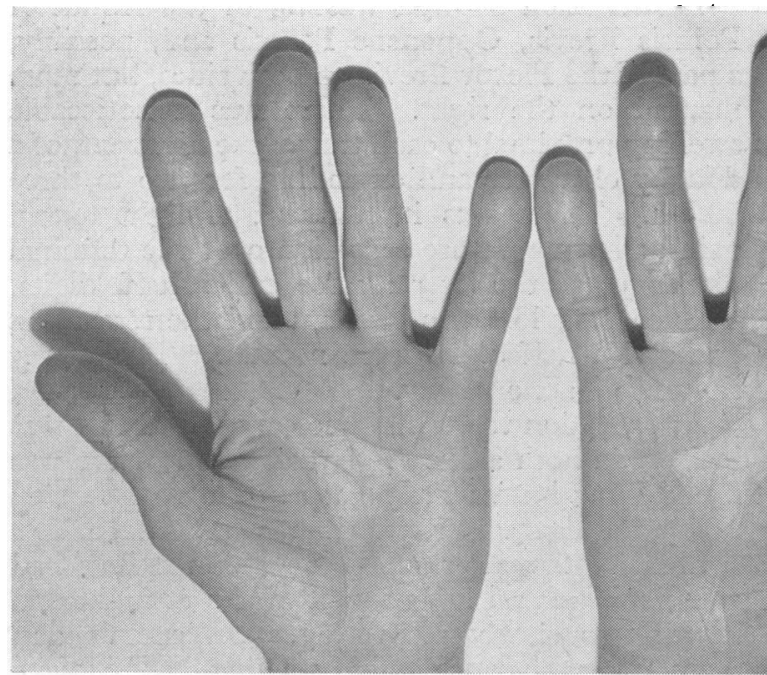

FIG. 3.-The hands of Patient B, showing gross wasting of the thenar eminences.
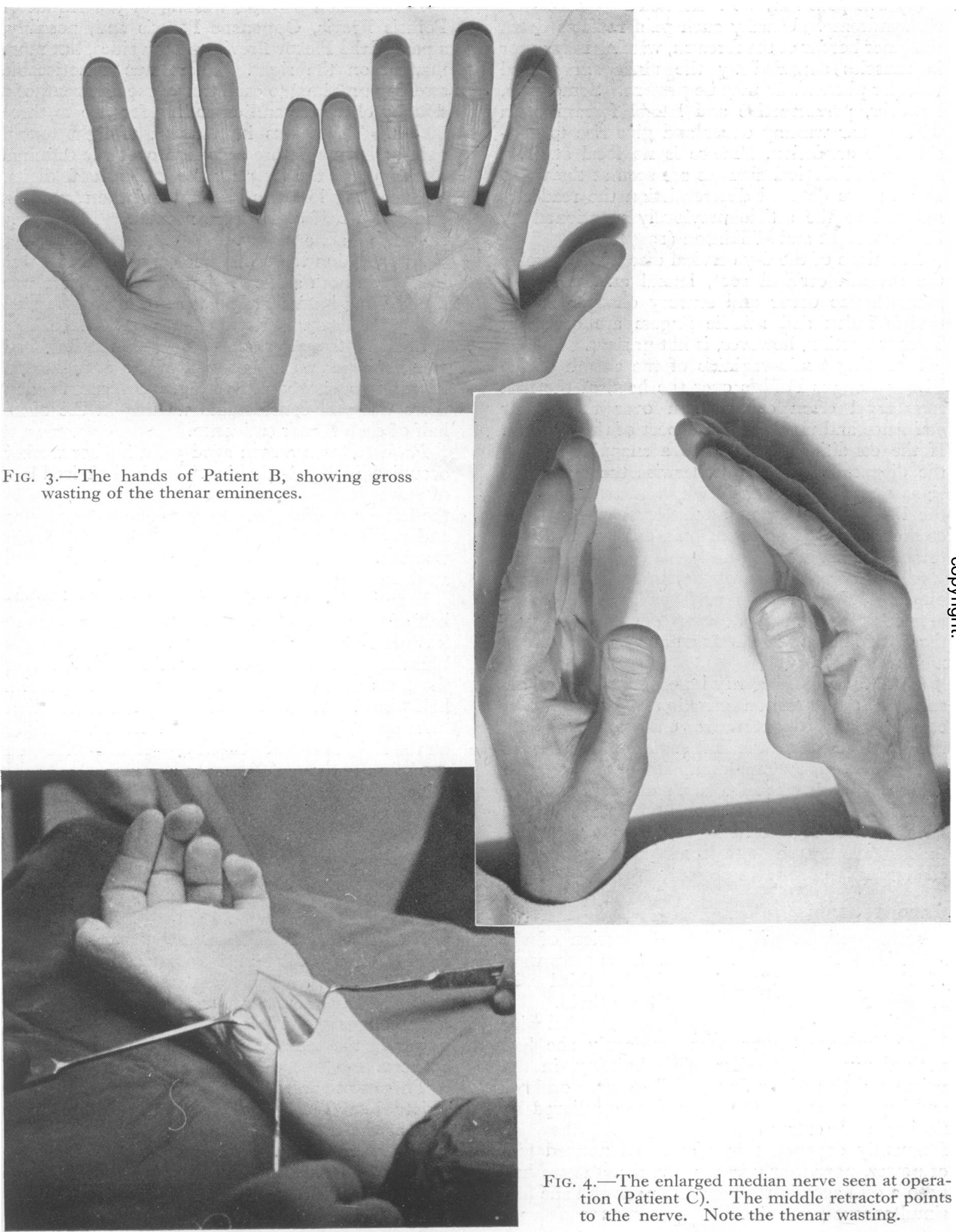

Fig. 4.-The enlarged median nerve seen at operation (Patient C). The middle retractor points to the nerve. Note the thenar wasting. 
(I) The pain may be simulated in the cervical rib syndrome. Usually such pain radiates down the inner border of the forearm, wasting may occur in muscles supplied by the ulnar nerve, and vascular phenomena may be present. Sometimes, however, paraesthesiae and lateral forearm pain with thenar wasting occur and give rise to considerable confusion. There is no local swelling and no radiological changes are seen at the wrist. For a more detailed differentiation the reader is referred to the article previously mentioned by Brain, Wright and Wilkinson (1947).

In lesions of the 6-7 cervical disc which involve the seventh cervical root, lateral arm pain and paraesthesiae occur and sensory changes in the thumb index and middle fingers are common. Thenar wasting, however, is not present.

Stenosing tendo-vaginitis of the sheath of the Flexor Longus Pollicis over the head of the first metacarpal often causes pain over the thenar eminence and up the flexor aspect of the forearm. If the condition advances to a snapping thumb the diagnosis is clear. Otherwise the only local signs may be slight nodularity and tenderness in the line of the tendon at the level of the basal crease of the thumb, with pain on flexion of the thumb against resistance.

(2) Thenar wasting may be simulated by lower motor neurone lesions such as anterior poliomyelitis. Such wasting, however, extends beyond the outer half of the thenar eminence and there is no sensory loss.

(3) A local swelling may be produced by lesions such as the common ganglion arising from a carpal joint; degenerative changes along the sheath of a deep tendon such as the Flexor Pollicis Longus ; compound palmar ganglion, and rare local conditions such as a parosteal lipoma or small haemangioma. It is most unusual, however, for any of these conditions to be associated with thenar wasting, though some median sensory changes may occur as a pressure effect.

\section{Case Reports}

Three patients with slow compression of the nerve have come under my care. Two cases were of the spontaneous type and the third was secondary to an old fracture of the scaphoid.

Patient $A$ was a woman aged 66 , occupied with house duties. Six months previously she had noticed pins and needles while knitting, in the palms and tips of the fingers. Pain came on later and kept her awake at night ; it was relieved by flexing and extending the fingers. She had frequently dropped things and had noticed loss of power, particularly in the thumbs. There had been a dull ache in the arm and neck and she had simultaneous headache.
Examination showed wasting of the Abductor Pollicis Brevis, Opponens Pollicis and, possibly, ㅡㅡ a part of the Flexor Brevis on both sides, but more $\frac{\pi}{\infty}$ marked on the right. There was a noticeable $\propto$ swelling proximal to each transverse ligament, of $\mathrm{a}^{\mathrm{C}}$ doughy character and extending for two to three $\overrightarrow{\vec{F}}$ inches. There was impairment of light touch, $\overrightarrow{0}$ pain and temperature sensation over the terminal phalanges of the lateral three and a half digits. This sensory loss was bilateral and the remaining $\widehat{\varnothing}$ one and a half digits were normal. The X-rayo showed no abnormality.

At operation the transverse carpal ligament was. $\vec{\circ}$ incised on both sides. A large swollen nerve was $\overrightarrow{\vec{\omega}}$ found lying immediately deep to the deep fascia ; under the ligament iteself it was constricted.

Six months after operation the tingling had 3 . gone, she was no longer kept awake at night andin power was slowly recovering. On examination $\dot{\dot{\sim}}$ there was still considerable wasting in the outer $\mathbb{}$ half of each thenar eminence.

Patient $B$ was a man aged 59 , a leather worker $\stackrel{\mathscr{A}}{\oplus}$ occupied in hand-stitching. He had noticed loss음 of power in the right thumb for four years and in the left for six months. Recently he had had pins 3 and needles in his fingers, especially in cold wet weather. Pain in the fingers and arms kept him $\overrightarrow{0}$ awake at night.

Examination showed gross wasting of the outeri half of the right thenar eminence and to a slighty less degree on the left (Fig. 3). There was blunting of light touch and pain sensation over the terminal phalanges of the lateral three and $a \stackrel{\Phi}{\circ}$ half digits. The X-rays showed no abnormality. $\stackrel{\varrho}{\Rightarrow}$

At operation a grossly swollen nerve was fcund on the right side and a slight degree of swelling on the left. The carpal ligament was incised on both sides. Three months after operation there was no recovery of muscle power or wasting on either 3 side, but the tingling and night pain had gone.

Patient $C$ was a man aged 42 . Twenty years ago he sustained a fracture of the scaphoid. Sub- $\bar{\varnothing}$ sequently the proximal fragment showed aseptic necrosis and secondary arthritis developed in theo wrist. The proximal fragment was excised some $\triangle$ time later. For the past six months he had noticedo pain in the other palm and fingers of the right hand. The pain was worse at night and radiated. up the forearm. He complained of some loss of $N_{0}$ power in the thumb, but the pain was his major concern.

There was severe limitation of movement at the wrist joint. There was some wasting of the outer part of the thenar eminence. Movements of the $\stackrel{?}{+}$ fingers were normal. No obvious loss of sensa- 0 tion was detected in the fingers. The $\mathrm{X}$-rays ${ }_{0}$ showed marked arthritic changes in the wristo joint (Fig. 2). 
At operation the median nerve was found swollen above and constricted beneath the transverse ligament (Fig. 4). The ligament was incised. Three months after operation there were no pins and needles and no pain. Power had improved little but the patient was entirely satisfied.

\section{BIBLIOGRAPHY}

BRAIN, W. R., WRIGHT, A. D., WILKINSON, M. (1947), Lancet, I, 277 (see correspondence following, p. 387).

DENNY-BROWN, D., BRENNER, C. (1944a), Arch. Neurol. Psychiat., 5I, I, and 52, I.

ELLIOTT, F. A., and KREMER, M. (1945), Lancet, I, 4. HIGHET, W. B. (1943), I.ancet, $i, 227$.

\title{
MULTIPLE MYELOMATOSIS
}

\section{A Clinico-Pathological Review, with a Report of a Case of Myeloblastic Type}

\author{
By HaRwood Stevenson, M.D., M.R.C.P. \\ Visiting Physician, The Royal National Orthopaedic Hospital, Stanmore
}

\begin{abstract}
' Bear this disease in mind when treating pains of the limbs of an obscure and intractable character.' This was said by Thos. Blizard Curling, F.R.S., Surgeon to the London Hospital, to William Macintyre Ioo years ago. The quotation is from the paper in which Macintyre published at his leisure in $185^{\circ}$ the clinical history of a case which had been noted to have ' urine of high density containing much animal matter, but without dropsy or cerebral disturbance.' The animal matter had been found to have unusual properties. The urine clouded on heating, cleared on boiling and coagulated on cooling again. Furthermore, it did not coagulate with nitric acid until it had stood for an hour or two. Then the coagulum cleared on boiling but solidified again on cooling. This strange behaviour had already been reported by Dr. Bence-Jones in 1848 . It is of great interest to read the original papers including the still earlier description of the morbid anatomy of the same case by Dalrymple in 1845 . The combined reports of the clinician Macintyre, the pathologist Dalrymple and the biochemist Bence-Jones made this the most completely reported case at that time and have led to its designation as the first definite case of multiple myelomatosis.
\end{abstract}

Macintyre tells the story of his patient with the humanity and feeling which at that period had not been banished from scientific papers. The account is a model of accuracy and clarity from the moment when his patient while on holiday "vaulting out of an underground cavern' falls to the ground with great pain in the chest and severe dyspnoea-his first spontaneous fracture-to the day when the story is completed by the pathologist. The ribs, sternum and spine were found to be soft and brittle and the latter collapsed to such a degree that the bodies of the lumbar vertebrae were scarcely thicker than those of the cervical. Dalrymple found the tumours which had so largely replaced the bones to contain 'oval cells with a faint grey nucleus and a bright and distinct nucleolus.' He also described and illustrated with wood-cuts cells with two, three or four such nuclei. He commented that there was no new bone formation as in osteo-sarcoma. He differentiated these cells from pus cells by their power of self-reproduction and described them as ' nucleolated nuclear cells capable of reproducing their kind.' He thought the disease was truly malignant.

These three papers all refer to earlier reports of cases of Mollities Ossium, a term which covered a considerable group of conditions. Macintyre's references show the difficulty of accurate differential diagnosis without biochemical aids. $\mathrm{He}$ refers to the term 'Medullary Gout' said to have been used by Saillant of Paris in 1792 , which suggests $\mathrm{n})$ more than pains in the bones with hollow spaces. We are on firmer ground, however, when we come to Howshipp (1826).

He described a woman of 35 whose history lasted for six years with 'stooping rolling gait,' pain in the bones, tenderness of the ribs, spontaneous fractures of both femora and massive albuminuria of undetermined type. At necropsy the femora, pelvis, lower parts of the tibiae, bodies of lumbar and dorsal vertebrae, ribs and sternum, could all be cut freely. The cortical bone was in many places reduced to egg-shell thickness and the bones contained cavities filled mostly with soft material varying in colour from dark blood to liver. The combination of pain, fractures, the bones affected, their cavitation and the length of history make either hyper- 